Florida International University FIU Digital Commons

8-2-1999

\title{
Trip generation study of gasoline stations with convenience market, quick service restaurant, and car wash in Miami-Dade county
}

Elio Roberto Espino

Florida International University

DOI: $10.25148 /$ etd.FI15101315

Follow this and additional works at: https://digitalcommons.fiu.edu/etd

Part of the Civil Engineering Commons

\section{Recommended Citation}

Espino, Elio Roberto, "Trip generation study of gasoline stations with convenience market, quick service restaurant, and car wash in Miami-Dade county" (1999). FIU Electronic Theses and Dissertations. 3243.

https://digitalcommons.fiu.edu/etd/3243 


\author{
Miami, Florida
}

TRIP GENERATION STUDY OF GASOLINE STATIONS WITH CONVENIENCE MARKET, QUICK SERVICE RESTAURANT, AND CAR WASH IN MIAMI-DADE COUNTY

A thesis submitted in partial fulfillment of the requirements for the degree of

MASTER OF SCIENCE in CIVIL ENGINEERING by Elio Roberto Espino 
To: Dean Gordon Hopkins

College of Engineering

This thesis, written by Elio Roberto Espino, and entitled Trip Generation Study of Gasoline Stations with Convenience Market, Quick Service Restaurant, and Car Wash, in Miami-Dade County having been approved in respect to style and intellectual content, is referred to you for judgment

We have read this thesis and recommend that it be approved.

Jose Guerrier

Rene de Huelbes

Sylvan Jolibois, Major Professor

Date of Defense : August 2, 1999

The thesis of Elio Roberto Espino is approved.

Dean Gordon R. Hopkins

College of Engineering

Dean Richard L. Campbell Division of Graduate Studies

Florida International University, 1999 


\section{DEDICATION}

I dedicate this thesis to my wife Christina. Without her patience, understanding, support, and most of all love, the completion of this work would not have been possible. 


\section{ACKNOWLEDGEMENTS}

I would like to express my most sincere gratitude to my advisors, Dr. Sylvan C. Jolibois Jr., Dr. Jose Guerrier, and Rene de Huelbes, P.E., for their continuous support, encouragement and guidance. They have always been willing to provide advise whenever needed.

The traffic data, that made this project a reality, were collected by Hesham Elbadrawi and Diana Ospina of the Lehman Center for Transportation Research. I would also like to acknowledge the owners of the five sites from which data were collected for allowing us to install the traffic counters on their locations.

Finally, I wish to thank the Federal Highway Administration's Dwight David Eisenhower Transportation Fellowship Program for its financial support which made possible my attending graduate school. 


\author{
ABSTRACT OF THE THESIS \\ TRIP GENERATION STUDY OF GASOLINE STATIONS WITH \\ CONVENIENCE MARKET, QUICK SERVICE RESTAURANT AND CAR \\ WASH IN MIAMI-DADE COUNTY \\ by
}

Elio Roberto Espino

Florida International University, 1999

Miami, Florida

Professor Sylvan C. Jolibois Jr., Major Professor

The objectives of the thesis are twofold: (a) validation of trip generation rates for land use 846 (LU-846) published in ITE's Trip Generation Manual, and (b) the generation of trip generation models for a newly introduced land use, the gasoline station with convenience market, quick service restaurant and car wash. Data was collected at five sites representative of the new land use and the number of trips produced by the sites were compared with estimates made using the ITE rates for LU-846. The ITE rates were found to underestimate trip generation by $50 \%$ to $400 \%$. Additionally, Multiple regression was performed using average daily trips and trips generated during the PM peak of the adjacent street as dependent variables and gross floor area of the convenience store and number of driveways as the independent variables. The models that resulted from the regression analysis are as follows: PMTRIPS $=82.75+0.0537 \mathrm{GFA}+7.23 \mathrm{DRW}, \mathrm{R}^{2}$ of 0.84 , and DTRIPS $=2135.7+0.239 \mathrm{GFA}+381.4 \mathrm{DRW}, \mathrm{R}^{2}$ of 0.70 . 


\section{TABLE OF CONTENTS}

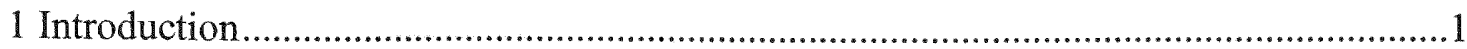

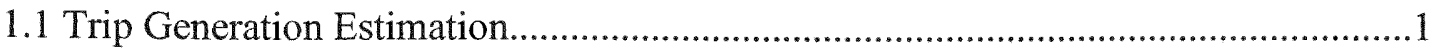

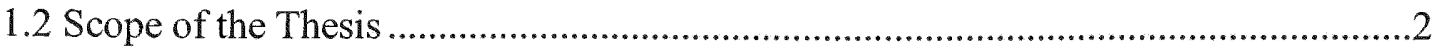

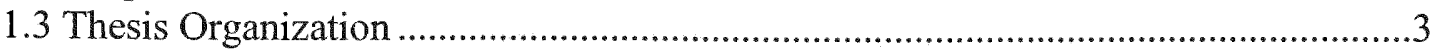

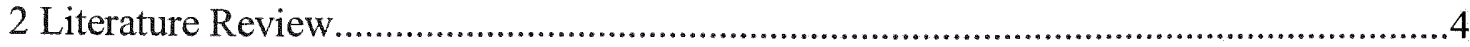

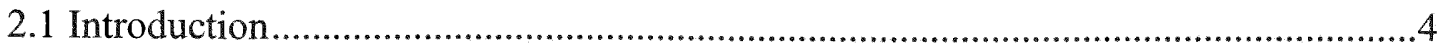

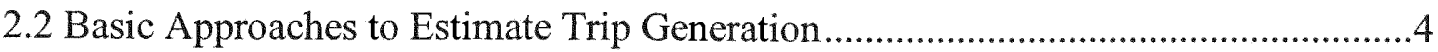

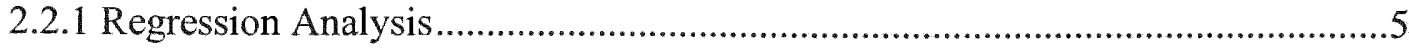

2.2.2 Assumptions and Limitations of the Linear Regression Model............................6

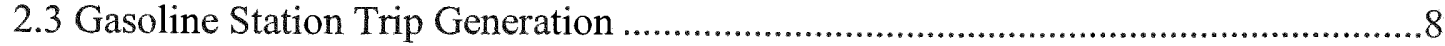

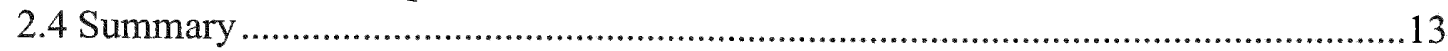

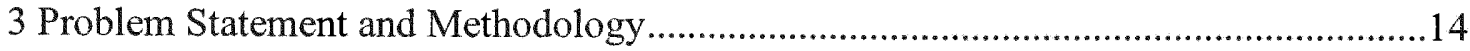

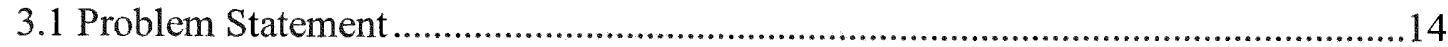

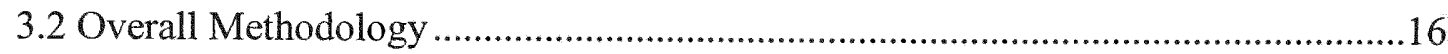

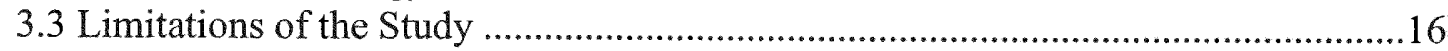

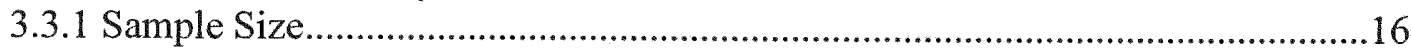

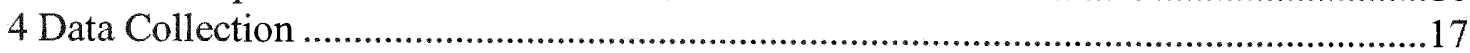

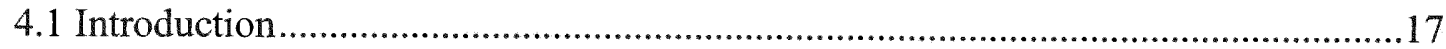

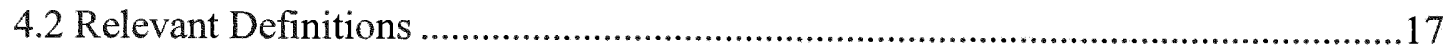

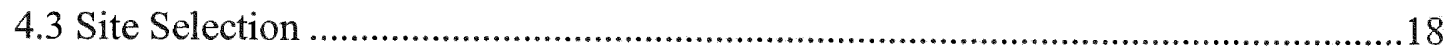

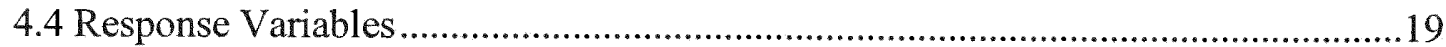

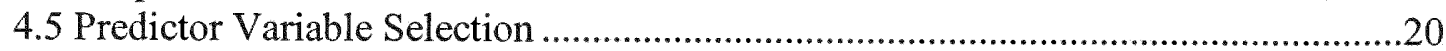

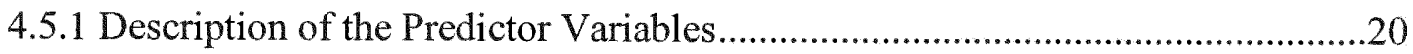

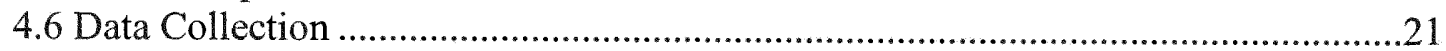

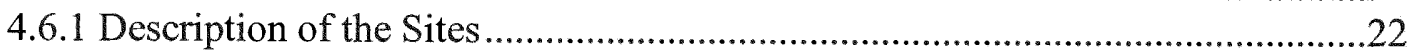

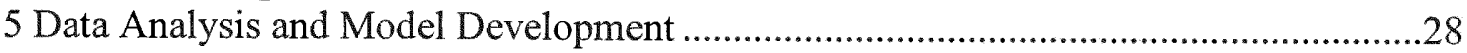

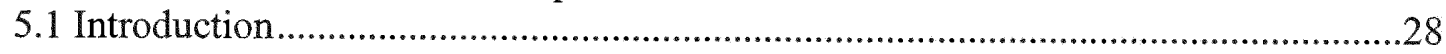

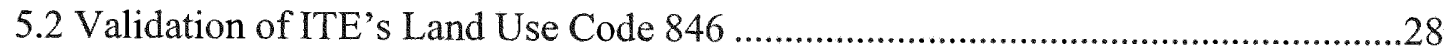

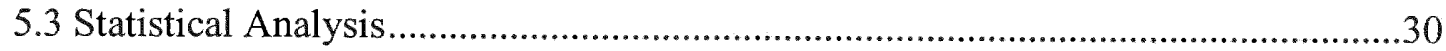

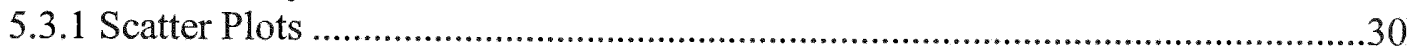

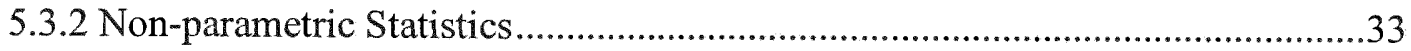

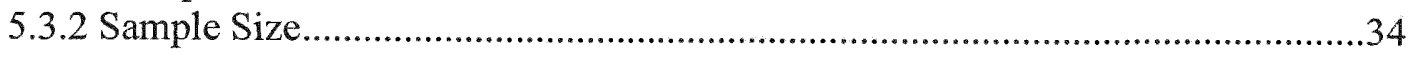

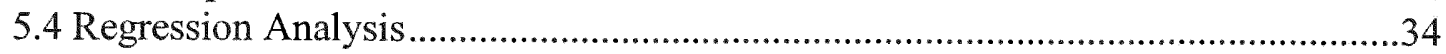

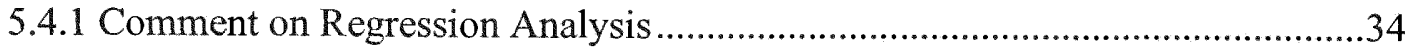

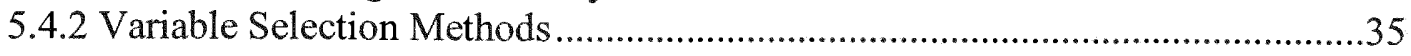

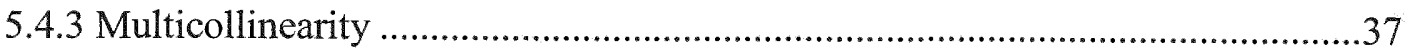

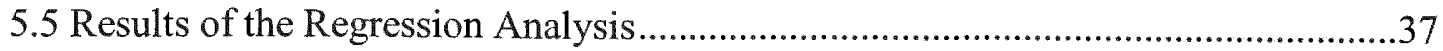

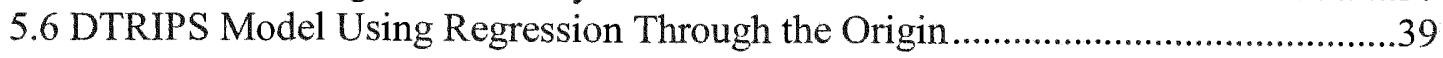

5.6.1 Comment on Regression Through the Origin .................................................40

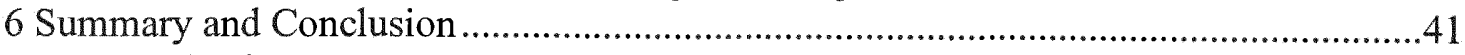

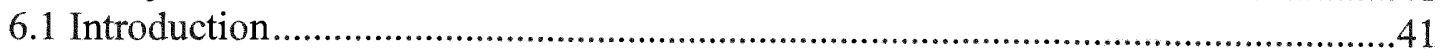


6.2 Discussion

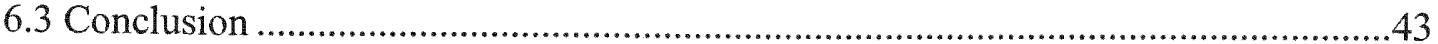

List of References 
4.1 Site 1: Amoco/Miami Subs Express ......................................................................23

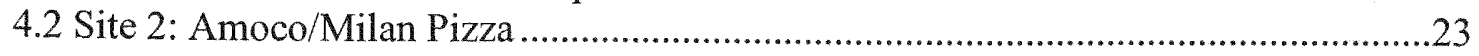

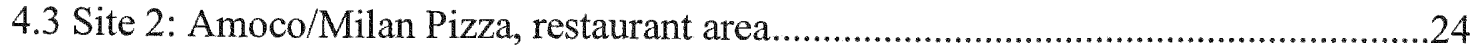

4.4 Site 3: Chevron/Subway/Dunkin Donuts..................................................................2. 24

4.5 Site 3: Subway restaurant, inside the convenience store .........................................25

4.6 Site 3: Seating area for Subway and Dunkin Donuts..............................................25

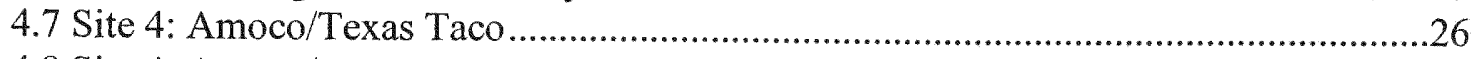

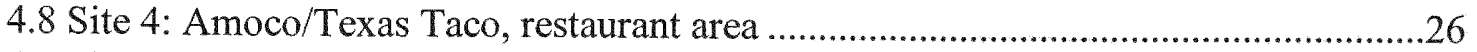

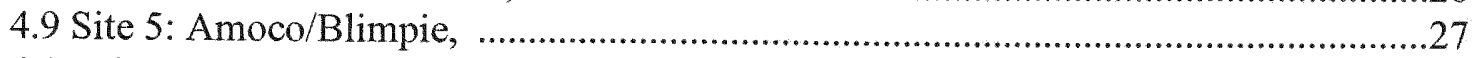

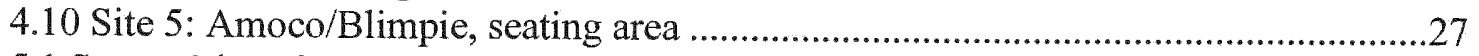

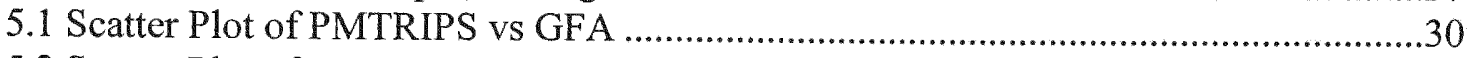

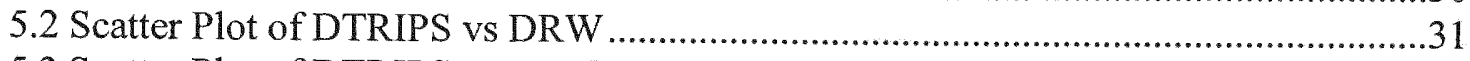

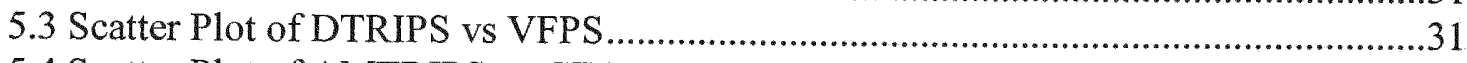

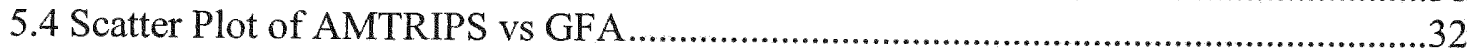

5.5 SPSS Output of Spearman's tho Correlation Coefficients ..........................................33

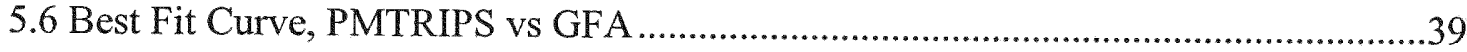




\section{CHAPTER 1}

\section{INTRODUCTION}

\subsection{Trip Generation Estimation}

The process of estimating the number of trips generated by new urban development is extremely important in transportation planning and traffic engineering. For example, developers determine site access requirements for proposed developments based on trip generation estimation. Moreover, traffic engineers use trip generation data to estimate future traffic volumes upon which off-site transportation improvements are based. Trip generation also provides guidance to communities to evaluate the implications of requests for zoning changes or of potential land use changes, (Trip Generation Handbook: An ITE Proposed Recommended Practice, 1998).

In Miami-Dade County, developer's fees are based in part on potential additional traffic to be generated by the proposed development. Applicants must submit a traffic impact study, in order to assess these impacts. The Florida Department of Transportation also requires a traffic study, which includes trip generation, or a trip generation study for new developments requesting access to the state road system. The study is used by FDOT to evaluate compliance with its access management guidelines. Therefore, accurate trip generation estimation plays an important role at the state and county levels in transportation planning and traffic engineering.

The Institute of Transportation Engineers publishes Trip Generation, which provides trip generation rates for different land uses. This widely used reference manual 
is based on hundreds of trip generation studies conducted nationwide. Trip Generation is intended to be a tool for planners, traffic engineers, zoning boards, and others in estimating the number of daily and peak hour trips generated on weekdays and weekends by a particular land use. Trip Generation is a dynamic document that has been expanded since it was first published in 1976. The latest edition, published in 1997, includes 19 more land uses than the previous edition, published in 1991. The new land uses incorporated in the publication are mainly the result of new types of developments introduced by developers, (Keller, Sherin and Connor, 1992).

The introduction of new land uses, which are not described in Trip Generation, requires the validation of the existing trip generation data or the development of trip generation models through a trip generation study. One new use introduced in the last three years in different markets of the United States including Miami-Dade County is the gasoline station with convenience market, quick service restaurant and a car wash.

\subsection{Scope of the Thesis}

The primary objectives of the thesis are two fold: (a) validation of trip generation rates for land use 846 (LU-846) published in ITE's Trip Generation Manual, and (b) the generation of trip generation models for a newly introduced land use, the gasoline station with convenience market, quick service restaurant and car wash. In order to validate LU846 and to develop the models, trip ends data were collected at five gasoline stations with convenience market, quick service restaurant and car wash in the Miami-Dade County area. Multiple regression analysis is used to build models that estimate the average daily trips and the PM peak hour trips of the new land use studied. The motivation for 
developing trip generation models for this new land use lies in the current lack of data for estimating trip generation for this type of development.

\subsection{Thesis Organization}

This thesis consists of six chapters. Following Chapter 1, Chapter 2 provides a review of the literature on trip generation and regression analysis. Chapter 3 outlines the problem statement, and describes the overall methodology for performing the multiple regression analysis. Chapter 4 describes the sites where the data were collected as well as the variables used in the analysis. Chapter 5 outlines the statistical models, and presents the results obtained through the use of the SPSS software. The thesis summary and conclusions are presented in Chapter 6. 


\section{CHAPTER 2}

\section{LITERATURE REVIEW}

\subsection{Introduction}

The objective of a trip generation study is to develop trip generation models that describe, with some level of confidence, the number of trips produced by a particular land use. Thus, trip generation modeling serves as a tool in the process of accurately assessing the impacts of new development on the existing transportation infrastructure. The strength of trip generation estimates is based on the correlation of some parameter of the proposed development, e.g. vehicle fueling positions, number of employees, or gross floor area with the amount of traffic generated by a site.

In the following sections, literature related to trip generation estimation of gasoline stations is reviewed. The review includes two parts: review of the basic methods of trip generation estimation and review of trip generation characteristics of gasoline stations.

\subsection{Basic Approaches to Estimate Trip Generation}

The Institute of Transportation Engineers' (ITE's) Trip Generation manual is recognized as the most authoritative source of trip generation information in the United States. This publication serves as the basis for establishing the amount of traffic generated by over 800 land uses. Trip generation rates published in Trip Generation are the result of data collected from trip generation studies conducted throughout the United States. 
The data analysis in this type of study is performed through regression analysis. In this analysis independent variables are chosen for the different types of uses. For instance, gross floor area, number of restaurant seats, and number of employees are some of the variables used by ITE. The confidence of the analysis rests primarily on the value of the coefficient of determination $\left(R^{2}\right)$. This coefficient provides a measure of the extend, the dependent variable is predicted by the independent variables. According to Ayyub and McCuen, (1996) the coefficient of determination equals the percentage of the variance in the criterion variable that is explained by the predictor variable. Because of this physical interpretation, $R^{2}$ is a meaningful indicator of the accuracy of the predictions. In trip generation the magnitude of $\mathrm{R}^{2}$ indicates the percentage in the variation of trips generated by a land use that can be attributed to any given independent variable or combination of variables if multiple regression analysis is performed.

\subsubsection{Regression Analysis}

Regression analysis refers to the methods by which estimates are made of the values of a variable, such as daily trips generated by a site. This is achieved from knowledge of the values of one or more other variables, and the measurement of the errors involved in this estimation process (Hamburg, 1985). The initial assumption is that a certain type of relationship between the variables exists. The linear model assumes that the dependent variable, $\mathrm{Y}$, can be characterized by a linear relationship with the independent variables plus an intercept. Thus the linear regression model has the form: 


$$
Y=\beta_{o}+\beta_{1} X_{1}+\beta_{2} X_{2}+\ldots+\beta_{n} X_{n}+\varepsilon
$$

Where:

$\mathrm{Y}$ is the response, or dependent variable.

$\beta_{0}, \beta_{1}, \ldots, \beta_{n}$ are unknown parameters.

$\mathrm{X}_{1}, \mathrm{X}_{2}, \ldots, \mathrm{X}_{\mathrm{n}}$ are regressors, or independent variables, as described above.

$\varepsilon$ is a random error term.

Regression analysis is used to find the values of $\beta_{0}, \beta_{1}, \ldots \beta_{n}$ which cause the above equation to "best fit" the observed data, where best fit is defined as minimizing the sum of squares of the differences between the observed and predicted variable.

\subsubsection{Assumptions and limitations Underlying the Linear Regression Model}

Freund and Minton, (1979) describe the assumptions and limitations of the linear regression model in the following manner: "The proper use of a linear regression model to analyze a set of data is subject to a number of assumptions. Failure of these assumptions and limitations may require the modification of the model or the placing of restrictions on the inference to be made by the analyses. Occasionally it may even lead to the abandonment of a regression analysis." Some of this assumptions and limitations presented by Freund and Minton, (1979) are outlined below.

- The model must adequately and correctly describe the data. This requirement does not mean that the model need not correctly describe the actual physical phenomenon 
underlying the data, but only that the model adequately describes the behavior of the data.

- The linear regression model must be linear in the parameters. However, the model may be nonlinear in the independent variables and yet remain within the framework of linear regression.

- The independent variables must be measured without error. If the independent variables are subject to error, the estimated regression coefficients may be biased. This bias is a function of the ratio of the measurement error of the model.

- The stated purpose of the regression model and analysis per se is to estimate the mean of the dependent variable for a specific set of values of the independent variable. Such estimation is performed within the range of the observed sample data and extrapolation is not recommended.

- The fact that a regression relationship has been found to exist does not imply a causeeffect relationship, although it may exist.

- The data on which the regression analysis is performed is assumed to be a random sample. However, regression analysis is often used in situations where this is not strictly the case.

Furthermore, Ayyub and McCuen (1996), state: "the principle of least squares assumes that the errors, that is, the differences between the predicted and measured values of the criterion variable, (1) are independent of each other, (2) have zero mean, (3) have a constant variance across all values of the predictor variable, and (4) are normally distributed. If any of these assumptions is violated we must assume that the model structure is not correct. Violations of these assumptions are easily identified using the 
statistical analysis of the residuals. When the sum of the residuals does not equal zero, it reflects a biased in the model. The regression approach, when applied analytically, requires the sum of the residuals for a linear model to equal zero."

\subsection{Gasoline Station Trip Generation}

The trip generation characteristics of gasoline stations, has been the subject of study by several researchers. Kawamura, 1993, conducted a comprehensive study of traffic, circulation, and parking of 30 service stations in different regions of the United States. These areas included Florida and other key markets. The study included companyoperated and dealer operated service stations of varying design. That is, with and without convenience marts, car washes, and service bays. The main objective of the study was to determine appropriate trip generation rates for service stations of varying design.

This study included as part of the field data gathering process, manual traffic and patronage counts for 12 and 14-hour periods. Additionally, 24-hour mechanized traffic counts were conducted on streets adjacent to each service station and at each driveway. These data were used to perform regression analysis with vehicle fueling positions as the independent variable. This analysis resulted in an $R^{2}$ of 0.32 between the number of VFPs and the total number of trips generated by the station.

Kawamura also compared the average daily trips produced by the study site and the actual counts. The ITE rates underestimated trip generation for service stations. But if the trip generation rates for other uses were combined to estimate the average daily trips produced by service stations with convenience marts and car washes, then the ITE rates overestimated the number of trips produced by the site. It is recommended in the study to 
combined single uses into one use in order to avoid overestimation of trip generation due to the consolidation of trips ${ }^{1}$. This research also concluded that the addition of a convenience mart, car wash, or some other type of use does not necessarily result in the same traffic increases that such uses would generate on their own as a separate development.

Datta, and Nannapaneni, (1998), conducted a similar study, which deals with the trip generation characteristics of gasoline/service stations in Michigan. The objectives of this research were to develop a database on trip-generating characteristics of multi-use developments, as well as create models that can be used for forecasting future trip generation characteristics for a proposed multi-use development. The type of multi-use development included in the study were gasoline stations with convenience stores and quick service restaurants. The combination of brands included Mobil/McDonalds, Shell/Taco Bell, Mobil/Subway, and Amoco/Subway/Dunkin Donuts. This study collected data at a total of 13 sites in Michigan. Eleven sites were used for model building and two sites were used for model validation. Analysis of the field data included the development of the mean and the standard deviation of the trip-end rates based on gross floor area.

The results of the field data analysis are as follows:

- A.M. Peak Hour: 43 mean trip ends per $1,000 \mathrm{ft}^{2}$ of Gross Floor Area (GFA) with a standard deviation of 24.99;

\footnotetext{
${ }^{1}$ Consolidation of trips refers to a service station customer using an ancillary service such as the convenience mart and car wash in one trip.
} 
- Midday Peak Hour: 49.75 mean trip ends per 1,000 $\mathrm{ft}^{2}$ of Gross Floor Area (GFA) with a standard deviation of 23.37 ;

- P.M. Peak Hour: 56.2 mean trip ends per $1,000 \mathrm{ft}^{2}$ of Gross Floor Area (GFA) with a standard deviation of 26.9 ;

Multiple regression analysis was also performed using the following independent variables: number of fueling positions, area of fast food, number of seats, and gross floor area. This analysis resulted in the following equations:

A.M. Peak period of the adjacent street

Trips $/$ hour $=(1.9945 \times N F P)+(0.015 \times A F F)+(1.5901 N S)+(0.0121 \times G F A)+42.5564$

Midday Peak period of the adjacent street

Trips $/$ hour $=(5.289 \times N F P)+(0.0105 \times A F F)+(2.9776 \times N S)+(0.0111 \times G F A)-55.3892$

$\underline{\text { P.M. Peak period of the adjacent street }}$

Trips $/$ hour $=(4.2642 \times N F P)+(0.0193 \times A F F)+(1.53 \times N S)+(0.0084 \times G F A)+44.4254$

The standard deviations and coefficients of determination $\mathrm{R}^{2}$ for the above models are:

A.M. peak: standard error $40.73, R^{2} 0.94$

Midday peak: standard error $38.68, \mathrm{R}^{2} 0.83$

P.M. peak: standard error $40.63, \mathrm{R}^{2} 0.83$

Where:

Trips/Hour $=$ trip ends generated by the site during the P.M. Peak Hour.

NFP = number of fueling positions

$\mathrm{AFF}=$ Area of Fast Food 
$\mathrm{NS}=$ Number of seats

GFA $=$ Gross Floor Area

As part of the data analysis, Datta, and Nannapaneni, did not calculate the average daily trips produced by the sites. This is significant since many agencies use daily trip generation in order to assess development fees. In this particular case only 11 sites were used for building the model and 4 independent variables were used to explain the variations in trips produced in the PM peak of the adjacent street. Multiple regression analysis with a low ratio of case-to-variable is not recommended since it tends to overestimate the degree of association of the variables. Tabachnick, B.G. and Fidell, L.S. (1983) refer to performing regression analysis with a low case to variable ratio in following manner: "One must have more cases than variables or the regression solution will be perfect and meaningless. With enough parameters, 'one can fit an elephant.' With enough variables, one can find a regression solution that completely predicts the dependent variable for all cases, but only as an artifact of the case-to-variable ratio. Ideally one would have 20 times more cases than variables. If stepwise regression is to be used, a procedure that is notorious for capitalizing on chance, a case-to-variable ratio of 40 to 1 would be appropriate. A suggested minimum requirement is to have at least 4 to 5 times more cases than independent variables. The lower the case-to-variable ratio, the more important it becomes that the residuals be normally distributed."

Finally, Long and Morrison, (1992) compiled data from five trip generation studies for convenience stores with gas pumps conducted in Montana and Florida. Linear regression was also used for the data analysis. The analysis performed showed a very poor correlation between the number of daily trips and vehicle fueling positions, $R^{2}$ of 
0.101. Better results were obtained, adjusted $R^{2}$ of 0.904 , with a multiple regression model using gross floor area, and vehicle fueling positions, as the independent variables, and trips during the PM peak of the adjacent street as the dependent variable. The resulting relationship was of the form:

$$
\text { SiteTrips }=0.0382323 \times G F A \times 15.9398 \times V F P
$$

The model presented above was obtained with a regression analysis through the origin. This type of linear relationship is called the no-intercept model and it is used when the regression line is known to go through the origin. In this case it implies that when GFA and VFP equal zero the site will not produce any trips. This assumption is questionable since it implies that trip generation only depends on the two independent variables chosen for the analysis, i.e. GFA and VFP. Furthermore, the $\mathrm{R}^{2}$ obtained with this type of analysis can not be compared with a coefficient of determination obtained with models that include an intercept. In this instance $\mathrm{R}^{2}$ measures the proportion of the variability of the predictor variable about the origin explained by the regression. Therefore the use of the no-intercept model makes it more difficult to establish the true relationship between the dependent and independent variables. Also it is important to point out that the degree of relationship between the independent variables may influence the overall $\mathrm{R}^{2}$ obtained. For instance GFA and VFP may highly correlated, therefore a model that includes both variables overestimates the degree of association between the dependent and independent variables. 


\subsection{Summary}

A review of the literature shows the use of simple regression models to estimate trip generation does not accurately explain the variations in trips produced by the sites. This is the result of the difficulty of finding one variable that can account for most of the variation in trip generation. For instance gross floor area and vehicle fueling positions have been used in simple regression and were found to account for approximately 10 to 30 percent of the variation in trips generated by the gasoline/service stations. These results are significant but not enough to assure accurate trip generation estimates since a large percentage of the variation in trips produced by the sites is not accounted for.

Choosing the independent variables to be used to build the models is extremely important. Therefore, predictor variables that may have been overlooked in the pass must be explored in order to build models that better explain the trip generation characteristics of the land use. The use of multiple regression was shown to improve the overall reliability of trip generation models. Multiple regression even though a powerful tool must be performed with relatively large sample size otherwise the analysis is meaningless. Sample size and careful consideration of the independent variables are paramount to the reliability of the analysis performed. 


\section{CHAPTER 3}

\section{PROBLEM STATEMENT AND METHODOLOGY}

\subsection{Problem Statement}

The introduction of new land uses, i.e. those not described in Trip Generation, requires the validation of the existing trip generation data or the development of trip generation models through a trip generation study. One new use is the gasoline station with convenience market, quick service restaurant and car wash introduced in different markets in the United States, including Miami-Dade County.

The Trip Generation manual includes land use codes LU-845, and LU-846, which are gasoline/service stations. These two land uses are described as follows:

Gasoline/Service Station with Convenience Market (845): Service stations are generally located at intersections or freeway interchanges. This land use includes service stations with convenience markets where the primary business is the fueling of motor vehicles, although they may have facilities for servicing and repairing motor vehicles. Some commonly sold convenience store items are newspapers, coffee, or other beverages, and snack items that are usually consumed in the car. This land use does not include stations with car washes.

Gasoline/Service Station with Convenience Market and Car Wash (846): Service stations are generally located at intersections or freeway interchanges. This land use includes service stations with convenience markets and car washes where the primary 
business is the fueling of motor vehicles, although they may also have facilities for servicing and repairing motor vehicles.

The two land uses described above are commonly found in Miami-Dade County. Also found in the Miami-Dade County area is a variation to LU-846. This type of gasoline station incorporates a quick service restaurant to the convenience market. This variation of LU-846 does not include a service station and therefore is significantly different from any land use described in ITE's Trip Generation manual. Moreover, the quick service restaurant found in this new use is not described in the ITE manual as a separate land use therefore trip generation can not be estimated by a combination of uses.

The rising popularity of this type of development presents a unique dilemma: how to estimate average trip ends, given the lack of available data. Permit records of the Florida Department of Transportation, researched for this study, found that the new sites developed in Miami-Dade County have estimated their trip generation using the rates for LU-846. Therefore it is imperative to collect local data in order to assess the validity of trip generation rates for LU-846 to estimate this new use. Additionally, the creation of trip generation models using local data is highly desirable. The creation of a local model will offer a higher degree of accuracy to estimate the number of trips produced by this emerging land use.

As reviewed in Chapter 2, a recent study conducted in Michigan analyzed the trip generation characteristics of similar sites. This study used multiple regression to generate a model to estimate the average number of trips during the PM peak of the adjacent street. Other research reviewed uses different methodologies for creating trip generation models. The research published up to date reflects the inherent difficulties in conducting 
this type of study, e.g. the challenges in finding an independent variable that can closely correlate with the number of trips generated by the site.

\subsection{Overall Methodology}

The methodology used in building this research closely follows the recommendations presented in the Trip Generation Handbook published by the Institute of Transportation Engineers for conducting a trip generation study. In following these guidelines data were collected at five gasoline stations with convenience market, quick service restaurant and car wash in Miami-Dade County. A rank order correlation matrix was created with the response and predictor variables in order to assess their degree of association. Regression analysis was performed on the variables with significant correlation in order to develop trip generation models for the average daily trips and trips during the PM peak hour of the adjacent street.

\subsection{Limitations of the Study}

\subsubsection{Sample Size}

As mention in chapter 2 the sample size is key when performing a multiple regression analysis. There are limitations in the number of independent variables that can be used given a particular sample size. Tabachnick, B. G. and Fidell recommend a minimum of four to five times more cases than independent variables. In this research two independent variables were used with a sample size of five, hence the results of the data analysis are constrained by this limiting factor. 


\section{CHAPTER 4}

\section{DATA COLLECTION}

\subsection{Introduction}

Obtaining reliable raw data was a major concern to realize this research. The accuracy of the data collected ultimately determines the validity of the results and conclusions of the analyses as well as the reliability of the models developed. In this section, the variables used in the regression analysis are defined. Additionally, a summary of the data collected, and a description of the sites surveyed are also presented in this chapter.

\subsection{Relevant Definitions}

It is important to define some parameters that help understand the data collected. Some of the definitions were taken from the Trip Generation Manual.

- Average Trip Rate: the weighted average of the number of vehicle trips or trip ends per unit of independent variable. The weighted average rate is calculated by summing all trips or trip ends and all independent variable units where paired data are available, and then dividing the sum of the trip ends by the sum of the independent variable units. 
- Average Trip Rate for the Peak Hour of the Adjacent Street Traffic: is the onehour weighted average vehicle trip generation rate at the site between 7 A.M. and 9 A.M. and between 4 P.M. and 6 P.M., when the combination of its traffic and the traffic on the adjacent street is the highest.

- A.M. and P.M. Peak Hour Volume of Adjacent Street Traffic: is the highest hourly volume of traffic on the adjacent street during the A.M. and P.M., respectively.

- Average Weekday Vehicle Trip Ends: is the average 24-hour total of all vehicle trips counted to and from a study site from Monday through Friday.

- Vehicle Fueling Position: is defined by the number of vehicles that can be fueled simultaneously at a service station.

\subsection{Site Selection}

The sites were selected based on the criteria recommended in the Trip Generation Handbook published by the Institute of Transportation Engineers. These guidelines are described below:

- The development should appear to be economically healthy

- The development should be mature (i.e., at least two years old) and located in a mature area so it represents the ultimate characteristics of a "successful" development.

- The data needed to describe the independent variables should be available.

- The site should be selected on the basis of the ability to obtain accurate trip generation and development characteristics. 
- It should be possible to isolate the site for counting purposes

- The site should consist of a single land use activity (unless a multi-use study is being conducted).

- There should be a minimal or no on-site construction or adjacent roadway construction.

Based on these guidelines, over 20 sites were identified in the Miami-Dade County area. From the sites initially surveyed, five sites were selected to collect trip generation data. An important factor that affected the site selection was the granting of approval for the data collection from the business owners. Approval was needed to install the pneumatic road tube counters at the access points to the gasoline stations. This type of device for recording traffic data was used because it allows the collection of data for continuous periods. Most of the owners contacted were reluctant to participate in the study. Furthermore, the sites selected were all located on major arterial, state roads.

\subsection{Response Variables}

Two response variables were studied. They are: average weekday traffic (DTRIPS), and trips generated during the PM peak hour of the adjacent street (PMTRIPS). These two response variables are the key determinant of the impact of the proposed development on the transportation infrastructure. The PM peak hour is considered instead of the AM peak hour following standard traffic engineering practice that assumes the highest combination of adjacent street, and site generated traffic during this period. Moreover, as mentioned in Chapter 2, average weekday traffic as well as PM peak hour trips are currently used by local agencies as a measure to assess permit fees. 


\subsection{Predictor Variable Selection}

The selection of the predictor variables underlies the assumption of a linear relationship between the response variable and the predictor variables. Moreover, the recommendations presented in the Trip Generation Handbook for the selection of the independent variables were considered. These guidelines are presented below:

- The data for the independent variable should be readily available, both for the survey site and for any potential proposed development of this land use type for which trip generation estimates may be desired.

- The number of trips generated at the site should be influenced in a logical way by the independent variable. Correlation does not equal causation.

- Available site data should be accurate, both for sites being counted and for proposed future development.

- Variables for similar sites should be provided directly and not be merely estimated from a different variable.

\subsubsection{Description of the Predictor Variables}

The predictor variables used in this study are:

- Vehicle Fueling Positions (VFPS): the number of vehicles that can be fueled simultaneously at a service station.

- Gross Floor Area (GFA): total area (in square feet) of the convenience store and quick service restaurant. This area includes any office space. 
- Daily Traffic of the Adjacent Street (DTADJST): the total weekday daily traffic of the adjacent street.

- AM peak hour traffic of the adjacent street (AMTADJ): traffic on the adjacent street during the AM peak hour.

- PM peak hour traffic of the adjacent street (PMTADJ): traffic on the adjacent street during the AM peak hour.

\subsection{Data Collection}

The trip generation data for the sites were collected using pneumatic road tube counters. Directional traffic volumes (entering and exiting) were counted by 15 -minute periods for a continuous 24-hour during a typical weekday, Tuesday, Wednesday, or Thursday. Additionally, during at least one weekend day, either Saturday, or Sunday traffic data was also counted. Moreover, the AM and PM peak hours of the adjacent street as well as the total daily traffic were obtained from the Florida Department of Transportation, Planning Office. A summary of all the data collected is depicted in table 4.1 below.

Table 4.1 Summary of Data Collected

\begin{tabular}{|c|c|c|c|c|c|c|c|c|c|}
\hline SITE & VFP & DTRIPS & GFA & AMTRIPS & PMTRIPS & AMTADJ & PMTADJ & DTADJST & DRW \\
\hline 1 & 8 & 4,625 & 4,500 & 240 & 382 & 889 & 1,061 & 20,000 & 4 \\
\hline 2 & 12 & 3,845 & 2,010 & 200 & 228 & 889 & 1,061 & 16,500 & 3 \\
\hline 3 & 8 & 5,018 & 3,875 & 293 & 290 & 4,370 & 1,705 & 26,500 & 4 \\
\hline 4 & 16 & 3,489 & 3,375 & 186 & 272 & 1,741 & 2,062 & 13,000 & 1 \\
\hline 5 & 12 & 3,492 & 3,300 & 311 & 264 & 1,600 & 1,221 & 13,000 & 3 \\
\hline
\end{tabular}




\subsubsection{Description of the Sites}

- Site one: Amoco/Miami Subs Express. This site is located at the intersection of Miami Gardens Drive, S.R. 860 and northwest $75^{\text {th }}$ avenue. State road 860 is classified as a minor arterial. Seating is available as well as an automated teller machine. This site offers a drive through window. There are three ingress/egress access drives to the site. Figure 4.1 on page 23 depicts site one

- Site two: Amoco/Milan pizza is located at the intersection of Miami Gardens Drive, (S. R. 860) and northwest $57^{\text {th }}$ avenue. Seating is not available. This site also offers an automated teller machine. There are three access drives to the site one is ingress only on Miami Gardens Drive the other two or both ingress/egress. Figures 4.2 and 4.3 on pages 24 and 25 respectively, depict site two.

- Site three: Chevron/Subway/Dunkin Donuts. This site is located on northwest 27 avenue, S.R. 9 is classified as a principal arterial. This site offers a drive through window and four driveways, three are ingress/egress and one is only egress. Figures $4.4,4.5$ and 4.6 in pages 25 and 26 depict site three.

- Site four: Amoco/Texas Taco. This site is located on northwest 119 street, S.R. 112. This state road is classified as a principal arterial. This site has a drive through window and seating is also available. This site also offers check-cashing services. There is only one ingress/egress access drive to the site. Figures 4.7 and 4.8 on page 27 depict site four.

- Site Five: Amoco/Blimpie. This site is located at the intersection of southwest 152 street and $116^{\text {th }}$ avenue. This site has three ingress/egress access drives. There is limited seating at this site. Figures 4.9 and 4.10 on page 28 depict site five. 


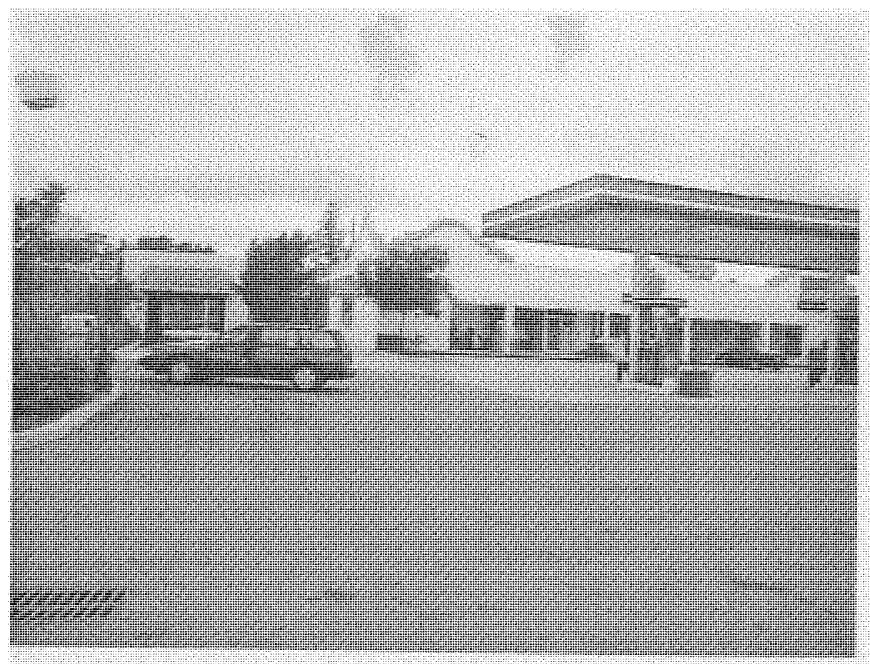

Figure 4.1: Site 1, Anoco/ Miami Subs Express

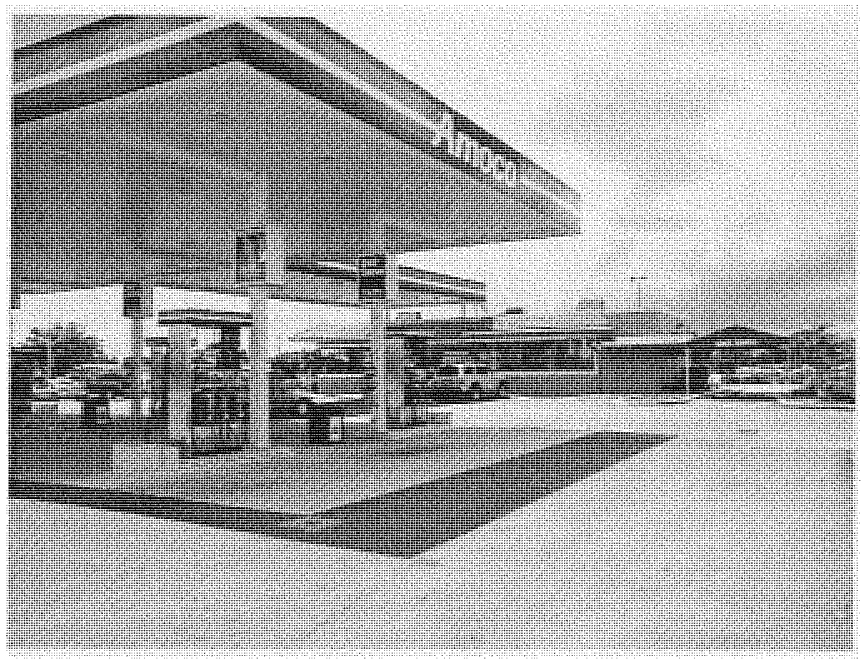

Figure 4.2: Site 2, Amoco/Milan Pizza 


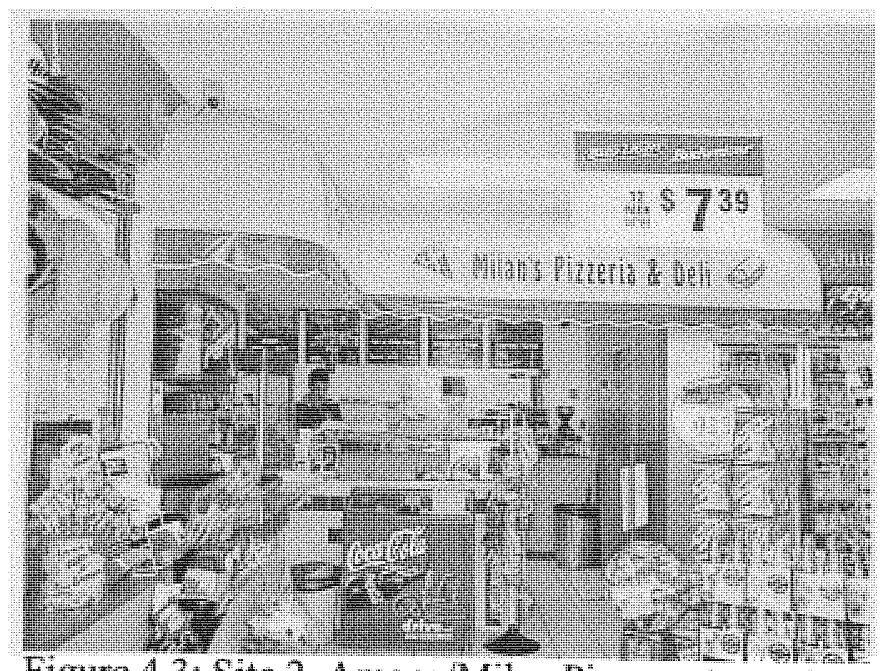

Figure 4.3: Site 2, Amoco/Milan Pizza testaurant area

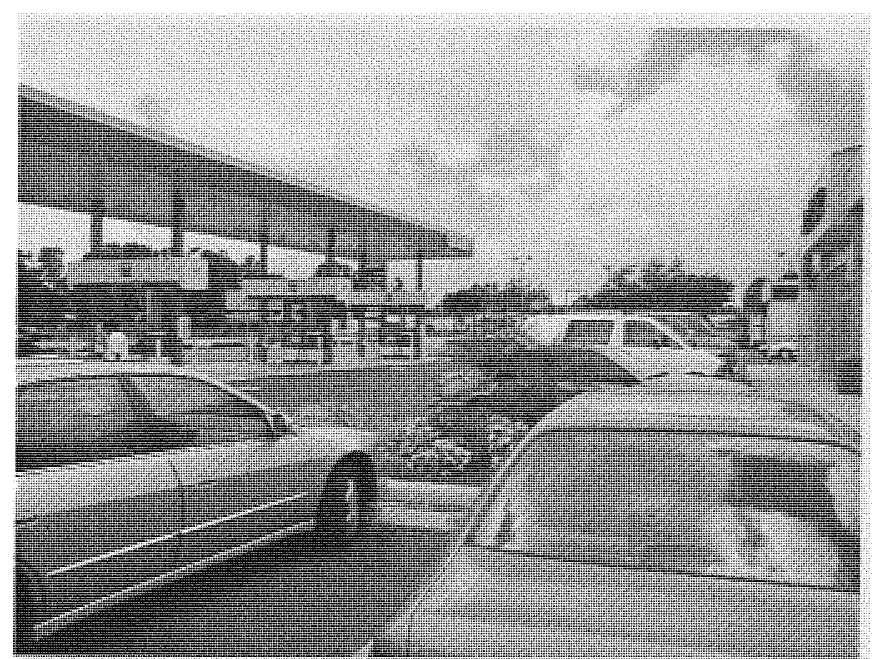

Figure 4,4: Site 3, Chevron/Subway/Dunkin Donuts 


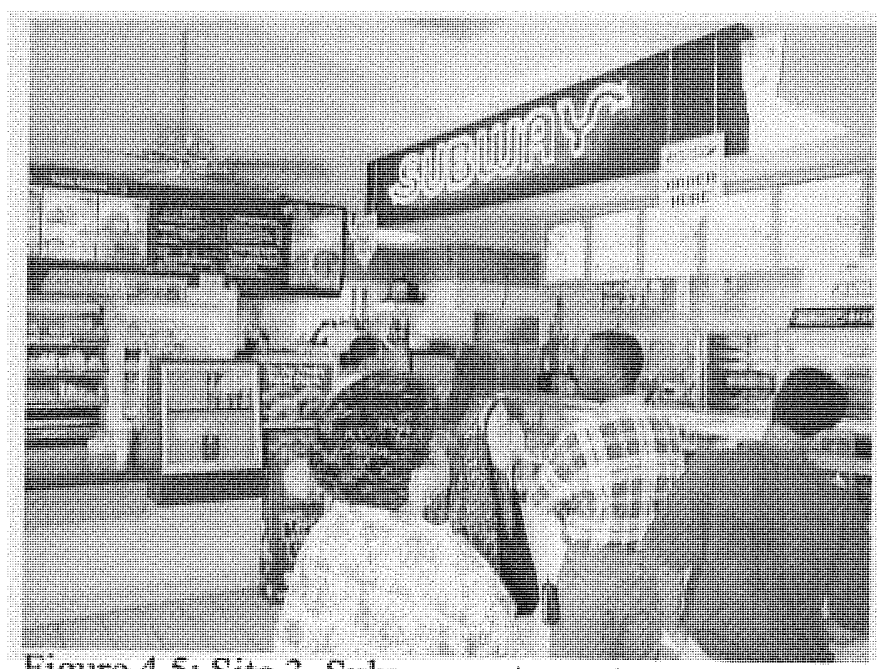

Figure 4:5: Site 3, Subway testurant

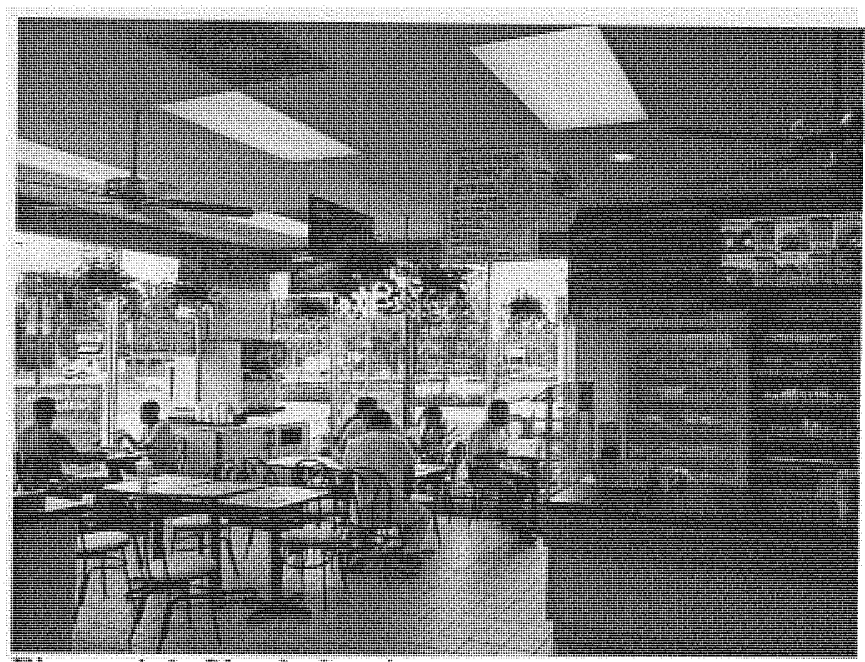

Figure 4.6: Site 3 , Seating area 


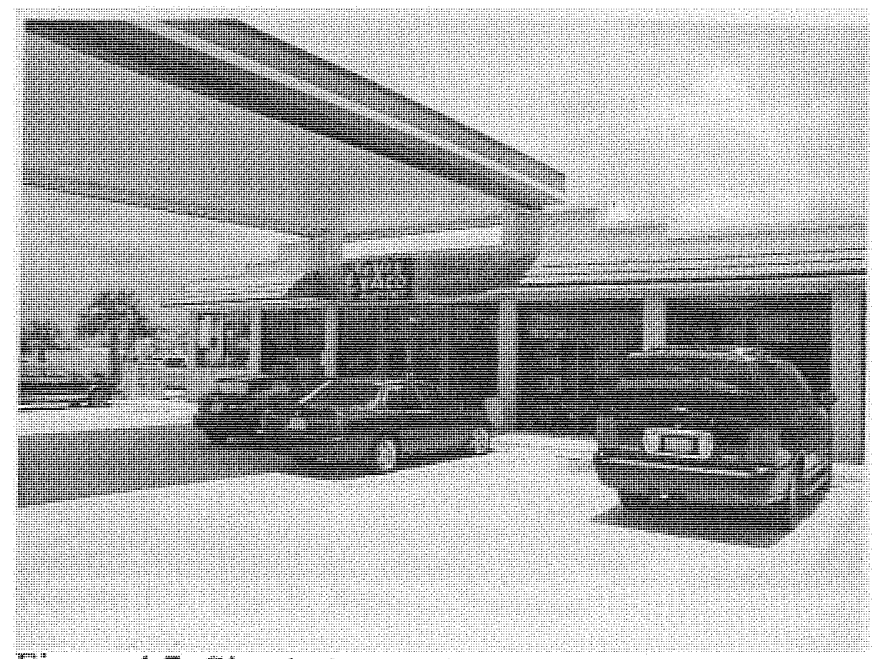

Figure 4.7: Site 4, Amoco/Texas Taco

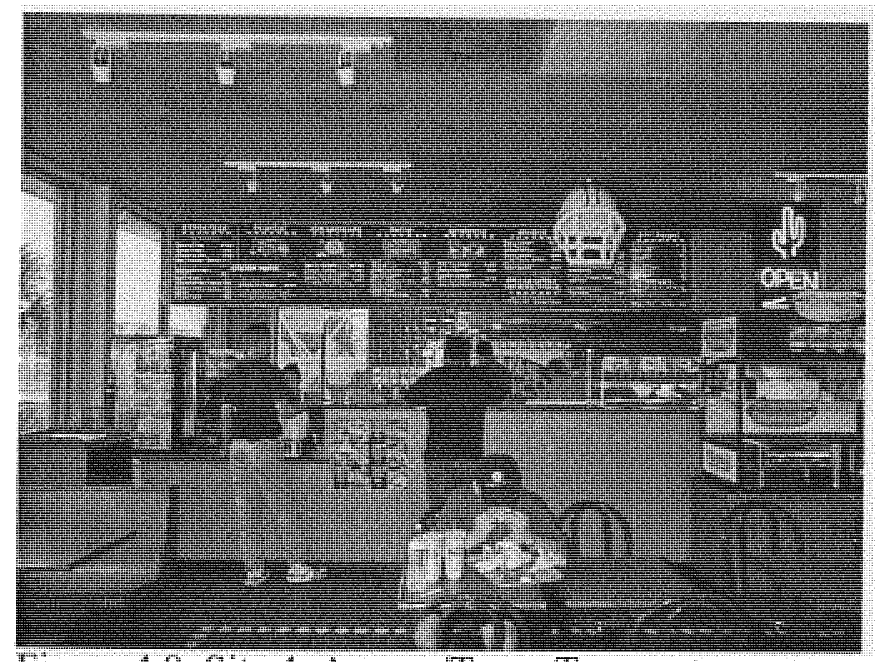

Figure 4.8: Site 4, Amoco/Texas Taco restaurant area 


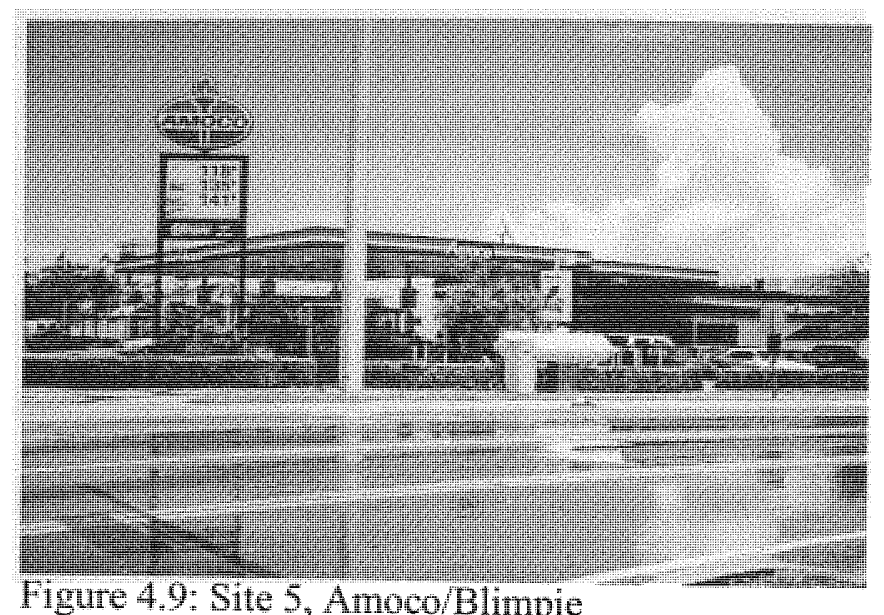

Figure 4.9: Site 5, Antoco/Blimpie

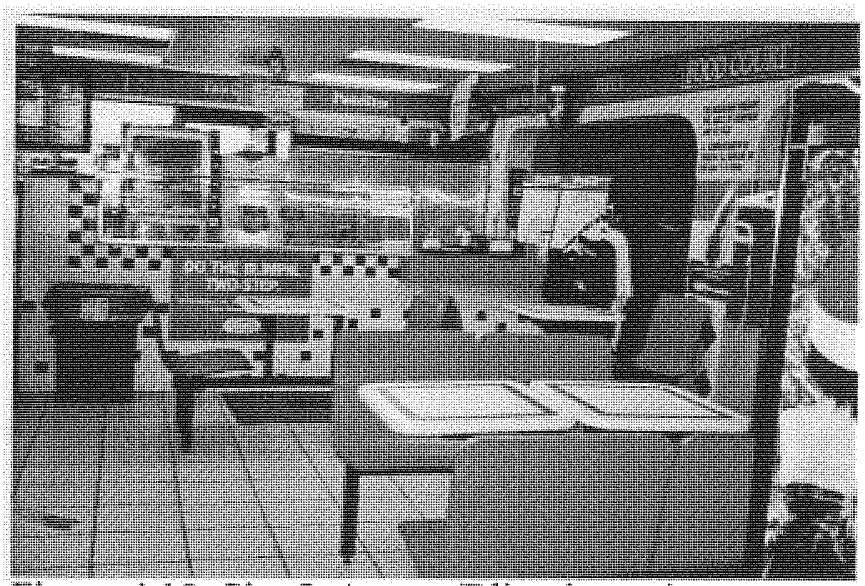

Figure 4.10: Site 5, Amoco/Blimpie seating area 


\section{CHAPTER 5}

\section{DATA ANALYSIS AND MODEL DEVELOPMENT}

\subsection{Introduction}

The statistical analyses of the data collected and the independent variables identified in chapter 4 are presented in this chapter. Also this chapter presents a brief comment on regression analysis and its relevance in the type analysis performed. In the first step of the data analysis, scatter plots were generated. This allowed visual examination of the extent to which the variables are related. Additionally, a rank order correlation was performed with the variables in order to assess their degree of relationship. The results of this analysis are presented in a correlation matrix. Additionally, regression analysis is performed on the response and predictors variables that exhibit the highest degree of correlation. The models are built from the regression analysis. All the statistical analyses were conducted using the latest edition, $9^{\text {th }}$ edition, of Statistical Package for the Social Sciences (SPSS).

\subsection{Validation of ITE's Land Use Code 846}

In this section estimates of the number of trips produced by the five sites surveyed are calculated using the ITE's trip generation rates, and then compared with the actual counts obtained at the sites. The Trip Generation manual uses vehicle fueling positions as the independent variable used to estimate trip rates for land use code 846 , gasoline/service station with convenience market and car wash. The trip rates presented in the ITE manual are trip ends on a weekday, during the AM and PM peak hours of the 
adjacent street and during the AM and PM peak hours of the generator. The coefficient of determination $\left(R^{2}\right)$ is not given, because its value is very low, i.e. the relationship between vehicle fueling positions and trips produced is not strong. Another variable or combination of variable may better explain the variation in trips produced by the sites. Table 5.1 below shows the values calculated with the ITE rates and the actual values collected at the sites.

\section{Table 5.1 ITE Rates VS Actual}

\begin{tabular}{|c|c|c|c|c|c|}
\hline $\begin{array}{c}\text { DTRIPS } \\
(\mathrm{ITE})\end{array}$ & $\begin{array}{c}\text { DTRIPS } \\
\text { (Actual) }\end{array}$ & $\begin{array}{c}\text { AMTRIPS } \\
\text { (ITE) }\end{array}$ & $\begin{array}{c}\text { AMTRIPS } \\
\text { (Actual) }\end{array}$ & $\begin{array}{c}\text { PMTRIPS } \\
\text { (ITE) }\end{array}$ & $\begin{array}{c}\text { PMTRIPS } \\
\text { (Actual) }\end{array}$ \\
\hline 1,223 & 4,625 & 85 & 240 & 106 & 382 \\
\hline 1,834 & 3,492 & 128 & 311 & 158 & 264 \\
\hline 1,834 & 3,845 & 128 & 200 & 158 & 228 \\
\hline 2,446 & 3,489 & 170 & 186 & 211 & 272 \\
\hline 1,223 & 5,018 & 85 & 293 & 106 & 290 \\
\hline
\end{tabular}

The ITE average trip ends were calculated with an average rate of 152.84 for DTRIPS, 10.64 for AMTRIPS, and 13.19 for PMTRIPS. The results of table 5.1 show the extreme discrepancy between the ITE rates and the actual counts. The ITE rates underestimated trip generation at the five study sites by $50 \%$ to $400 \%$. These results underlie the fact that the ITE rates for LU-846 are highly unreliable when used to estimate trips produced by gasoline stations with convenience market, quick service restaurant and car 
wash. Therefore the trip producing characteristics of this newly introduced land use must be further studied. Thus more accurate means of estimating trip generation may be found.

\subsection{Statistical Analysis}

\subsubsection{Scatter Plots}

Scatter diagrams provide an overall picture of the relationship between the variables used in the statistical analysis. Scatter plots also serve to test the strength of the relationship by the amount of scatter over a small range, but also by the constancy of the vertical scatter throughout the range of $\mathrm{X}$ values. Furthermore, an examination of a scatter diagram serves as an indication of whether a straight line appears to be an adequate description of the relationship between the two variables. Scatter plots of the variables PMTRIPS and GFA were generated as well as DTRIPS and DRW, DTRIPS and VFPS, and AMTRIPS vs GFA. These graphs are presented below.

\section{Figure 5.1 Scatter plot of PMTRIPS vs GFA}

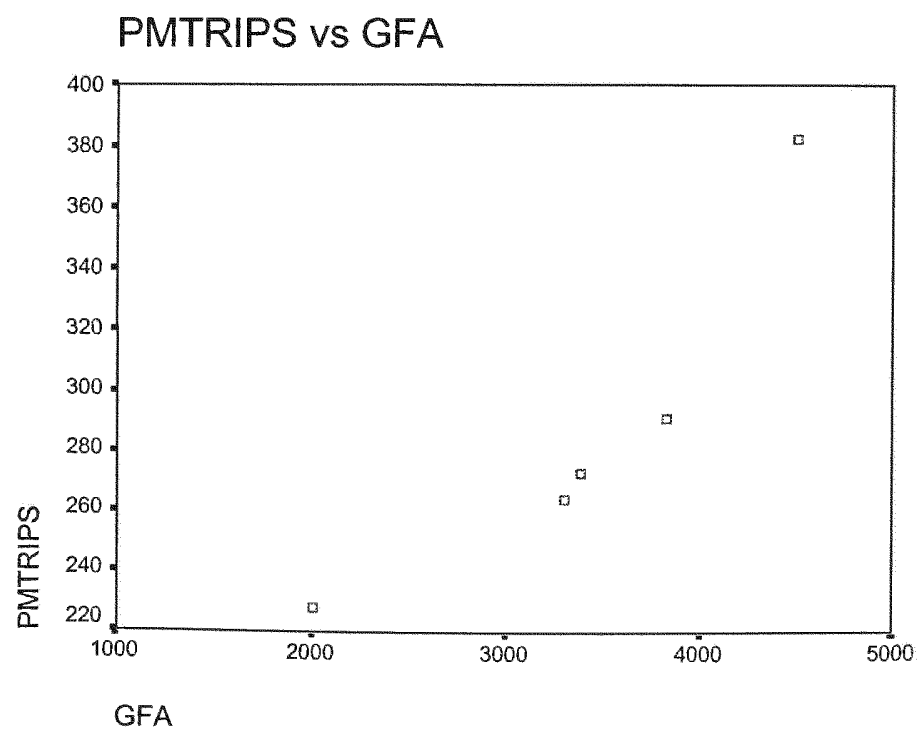


Figure 5.2 Scatter plot of DTRIPS vs DRW

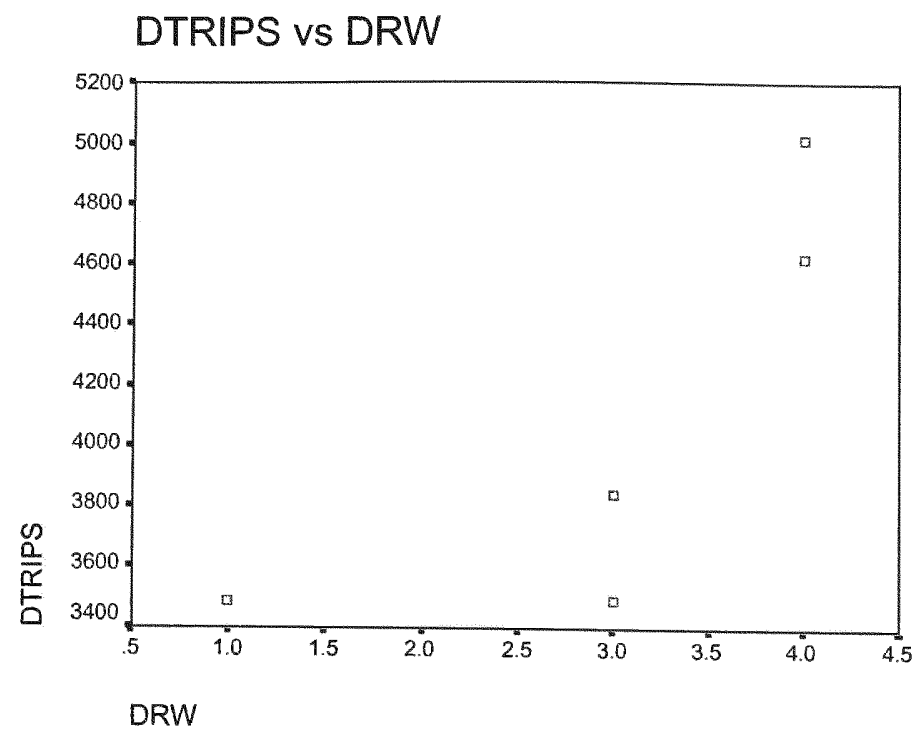

Figure 5.3 Scatter plot of DTRIPS vs VFPS

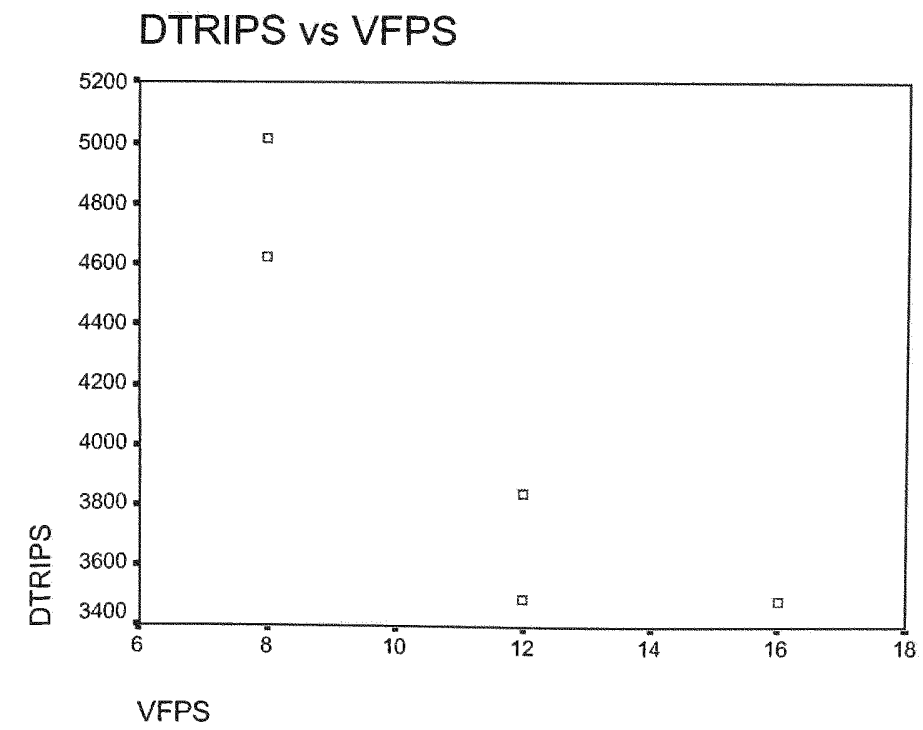


Abdel-Aty (2003) developed injury severity models for roadway sections, signalized intersections, and toll plazas using crash data for the Central Florida region (i.e., Orange, Osceola and Seminole Counties). The variables included in the model were related to the driver, vehicle and roadway. These variables are summarized in Table 2-3.

Table 2-3 Variables Included in Study by Abdel-Aty (2003)

\begin{tabular}{|c|c|}
\hline Variable Type & Variable \\
\hline \multirow{7}{*}{$\begin{array}{c}\text { Human Factors } \\
\text { (Driver Characteristics) }\end{array}$} & Driver's age \\
\hline & Age of other driver \\
\hline & Gender \\
\hline & Alcohol involvement \\
\hline & Point of impact of at fault driver \\
\hline & Harmful event \\
\hline & Use of seat belt \\
\hline \multirow{2}{*}{ Environmental } & Road Surface condition (wet or dry) \\
\hline & Lighting condition \\
\hline \multirow{8}{*}{$\begin{array}{c}\text { Roadway } \\
\text { and } \\
\text { Traffic Characteristics }\end{array}$} & Area type (urban or rural) \\
\hline & Presence of median \\
\hline & Pavement friction number \\
\hline & Lane width \\
\hline & Shoulder width \\
\hline & Access control type \\
\hline & Section AADT \\
\hline & Vehicle type \\
\hline
\end{tabular}

The results of the study indicated that driver's age, gender, seat belt use, point of impact, speed, and vehicle type were significant predictors of injury severity levels across all models. Furthermore, driver's violation, alcohol involvement, lighting condition, and the presence of a horizontal curve were positively correlated with injury severity risk. The ordered probit models developed for roadway sections and signalized intersections are summarized in Tables 2-4 and 2-5. 
scattered. A similar observation can be made of AMTRIPS vs GFA plot. Hence a linear relationship can not be established by the simple inspection of the scatter plot

\subsubsection{Non-parametric Statistics}

As mentioned in chapter 2 the linear model assumes a linear relationship between the variables. Non-parametric or distribution-free tests do not make assumptions about the underlying population. One such test is the Spearman's rho correlation or rank oder correlation. Since it was only possible to collect data at five sites this type of analysis is better suited in this case. The Spearman's rho correlation was used to estimate the degree of association between the variables studied. This type of analysis was used since The results of this analysis is presented in table 5.2 below

Figure 5.5 SPSS Output, Spearman's rho Correlation Coefficients

\begin{tabular}{|c|c|c|c|c|c|c|c|c|c|c|c|}
\hline \multicolumn{12}{|c|}{ Corrolations } \\
\hline & & & PMTRIPS & AMTRIPS & DTRIPS & GFA & VFPS & DRW & AMTADJ & PMTADJ & DTADJST \\
\hline \multirow[t]{27}{*}{ Spearmans tho } & \multirow[t]{3}{*}{ PMाRाएड } & Correlation Coefficient & 1.000 & .100 & .500 & $1.000^{*}$ & -.632 & .632 & .205 & .103 & .205 \\
\hline & & Sig. (2-talled) & & 873 & .391 & . & 252 & .252 & 741 & .870 & 741 \\
\hline & & $N$ & 5 & 5 & 5 & 5 & 5 & 5 & 5 & 5 & 5 \\
\hline & \multirow[t]{3}{*}{ AMIRIPS } & Comelation Coefficient & 100 & 1000 & .400 & .100 &. .527 & 527 & .154 & -154 & 154 \\
\hline & & Sig. (2-tailed) & 873 & & .505 & 873 & 361 & .361 & .805 & .805 & 805 \\
\hline & & $\mathrm{N}$ & 5 & 5 & 5 & 5 & 5 & 5 & 5 & 5 & 5 \\
\hline & \multirow[t]{3}{*}{ DTRIPS } & Comrelation Coefficient & 500 & 400 & 1.000 & 500 & $-949^{\circ}$ & $.949^{\circ}$ & .051 & .359 & 051 \\
\hline & & Sig. (2-talled) & 391 & .505 & & 391 & .014 & .014 & 935 & .553 & 935 \\
\hline & & $\mathrm{N}$ & 5 & 5 & 5 & 5 & 5 & 5 & 5 & 5 & 5 \\
\hline & \multirow[t]{3}{*}{ GFA } & Correlation Coefficient & $1.000^{+1}$ & .100 & 500 & 1.000 & .632 & 632 & .205 & .103 & 205 \\
\hline & & Sig. (2-talled) & . & .873 & 391 & . & .252 & .252 & .741 & 870 & .741 \\
\hline & & N & 5 & 5 & 5 & 5 & 5 & 5 & 5 & 5 & 5 \\
\hline & \multirow[t]{3}{*}{ VFPS } & Correlation Coefficient & -.632 & -.527 & $.940^{\circ}$ & .632 & 1.000 & $-1.000^{-1}$ & .054 & 433 & .054 \\
\hline & & Sig. (2-talled) & .252 & .361 & .014 & .252 & & .000 & 931 & 467 & .931 \\
\hline & & $\mathrm{N}$ & 5 & 5 & 5 & 5 & 5 & 5 & 5 & 5 & 5 \\
\hline & \multirow[t]{3}{*}{ DRW } & Correlation Coefficient & 632 & .527 & $949^{*}$ & 632 & $-1.000 \times+$ & 1.000 & $-.05 \%$ &,- 433 & .054 \\
\hline & & Sig. (2-talled) & 252 & 361 & .014 & .252 & .000 & . & .931 & .467 & 931 \\
\hline & & N & 5 & 5 & 5 & 5 & 5 & 5 & 5 & 5 & 5 \\
\hline & \multirow[t]{3}{*}{ AMTADJ } & Correlation Coefficient & 205 & .154 & .051 & .205 & .054 & .054 & 1.000 & $895^{\circ}$ & $1.000^{\prime \prime}$ \\
\hline & & Sig. (2ntailed) & .744 & .805 & 935 & .741 & .931 & .931 & & .040 & . \\
\hline & & $\mathrm{N}$ & 5 & 5 & 5 & 5 & 5 & 5 & 5 & 5 & 5 \\
\hline & \multirow[t]{3}{*}{ PMTADJ } & Correlation Coefficient & 103 & -.154 & -.359 & 103 & .433 & -433 & $895^{6}$ & 1.000 & $895^{\circ}$ \\
\hline & & Sig (2-tailed) & 870 & .805 & .553 & .870 & .467 & .467 & .040 & & .040 \\
\hline & & $N$ & 5 & 5 & 5 & 5 & 5 & 5 & 5 & 5 & 5 \\
\hline & \multirow[t]{3}{*}{ DTADJST } & Correlation Coefficient & 205 & .154 & .051 & 205 & .054 & 9.054 & $1.000^{\circ}$ & $.895^{\circ}$ & 1.000 \\
\hline & & Sig. (2-ailed) & 741 & 805 & .935 & .741 & .931 & .931 & . & .040 &. \\
\hline & & N & 5 & 5 & 5 & 5 & 5 & 5 & 5 & 5 & 5 \\
\hline
\end{tabular}


As shown in the table above the PMTRIPS and GFA are highly correlated with a correlation coefficient of 1 . There was also a significant correlation, 0.949 , between DTRIPS, average daily trips and DRW or access points. It is also relevant to point out the perfect inverse linear relationship between VFP and DRW.

\subsubsection{Sample Size}

The accuracy of a model tends to improve with increases in sample size. In this study data were collected from five sites since they were the only ones from the 20 sites identified which gave us permission to collect data on their premises. While small this sample size is congruent with common practice in the traffic planning industry to collect data at three to five sites that truly meet the recommended site selection criteria. It is assumed data collected from such samples will be relatively stable (Trip Generation Handbook, 1998).

\subsection{Regression Analysis}

\subsubsection{Comment on Regression Analysis}

Regression analysis is a statistical tool that utilizes the relation between two or more quantitative variables so that one can be predicted from the other, or others (Neter, Wasserman and Kutner, 1983). Regression analysis with one independent variable is called a simple regression and when two or more independent variables are used to predict the values of the dependent variable the analysis is called multiple regression. As mentioned in chapter 2, the assumptions of linear regression are the following: the differences between the predicted and measured values of the criterion variable, (1) are 
independent of each other, (2) have zero mean, (3) have a constant variance across all values of the predictor variable, and (4) are normally distributed (Ayyub and McCuen, 1996).

\subsubsection{Variable selection Methods}

When performing multiple regression an important consideration is the number of predictor variables that will be included in the model. It is common practice to consider for inclusion a large number of variables, then select a subset of variables or terms which appear most relevant, and finally to specified the model on the basis of the selected terms. This does not constitute a strictly valid statistical procedure, since the correctness of the type I and type II error probabilities associated with statistical hypothesis tests assumes that a single model and a restricted set of hypotheses have been specified prior to data collection. In other words, the model and associated hypothesis should not be determined by the data (Freund and Minton, 1979).

However, the initial inclusion of a large number of variables may be justified by the fact that the omission of essential variables may produce biased estimates while the inclusion of extraneous variables does not. Furthermore, much research is of an essentially exploratory nature, thus precluding the correctly specified models. For these reasons statisticians have developed a number of variable selection procedures.

The variable selection procedures are formulated with these two questions in mind:

1. How many variables should be included in the model? 
2. Given the number of variables to be included, what specific subset of variables provides the minimum residual mean square?

These aspects are highly related and most variable selection procedures combine them. The procedures that have been widely used are:

- Backward Elimination. In this procedure, an initial analysis is performed using all variables calculated. The t-statistic for significance of each coefficient is calculated and the regression coefficient with minimum absolute t-value, i.e. the "least" significant coefficient is deleted. The remaining variables produce an equation with one less coefficient. All t-statistics are computed for the new regression and again the least significant coefficient is deleted, etc. This procedure is a one-at-a-time elimination, or a step-down procedure.

- Forward Selection. In contrast to a backward elimination, this procedure starts with the "best" one-variable equation and adds additional variables, one at a time. Hence, it is sometimes called a step-up procedure. The independent variable for the best single-variable equation is obtained by seeking the maximum of the absolute pairwise correlations of the dependent and independent variables. The second is chosen by seeking the maximum of the partial correlations of the remaining independent variables with the dependent variable, adjusted for the variable already chosen.

- Stepwise. This procedure is a refinement of the forward selection procedure. At each step, before the determination of the next variable to be added, the statistics for significance of the already chosen coefficients are examined to see if a variable elimination may be in order. This procedure can thus be described as a step-up 
procedure with a step-down adjustment. The procedure stops when, for specified significance levels, neither a forward selection nor a backward elimination is indicated.

\subsubsection{Multicollinearity}

In multiple regression analysis, it is important to consider the nature and significance of the relations between the independent variables and the dependent variable. Another major concern is the correlation of the independent variables among themselves. This is called intercorrelation or multicollinearity (Neter, Wasserman and Kutner, 1983). When independent variables are correlated, the regression coefficient yielded by the relationship of any independent with the dependent variable depends on which other independent variables are included in the model and which ones are left out. Thus, a regression coefficient does not reflect any inherent effect of the particular independent variable on the dependent variable but only a marginal or partial effect, given whatever other independent variables are included in the model.

\subsection{Results of the Regression Analysis}

Multiple regression analysis was performed using the variables that showed the highest correlation values. The dependent variables used were average trips generated during the PM peak hour of the adjacent street (PMTRIPS) and average daily trips (DTRIPS). The independent variables used in the analysis were gross floor area of the convenience store (GFA) and number of driveways (DRW). Multiple regression with two variables was performed even though data was available for only five sites. This was 
done in order to assess the individual contributions of the two variables to the models. The two models generated are presented below:

$$
\text { PMTRIPS }=82.75+0.0537 G F A+7.23 D R W \quad ;
$$

$$
D T R I P S=2135.7+0.239 G F A+381.4 G F A \quad ;
$$

The standardized coefficients for the PMTRIPS model are .851 for GFA and .154 for DRW. Therefore, GFA has significantly more weight in the model than DRW. The coefficient of determination, $\mathrm{R}^{2}$, for this model is .84 . Therefore, $84 \%$ in the variation of PMTRIPS, can be explained by the independent variables. For the second model (DTRIPS) the standardized coefficients are .315 for GFA and .673 for DRW. In this case the results show a greater weight of DRW than GFA. The coefficient of determination for this model is .70 . The overall significance level for the first model is 0.16 and for the second 0.30

Furthermore a simple regression equation was generated using PMTRIPS as the independent variable and GFA as the independent variable. This analysis was performed given the high correlation found between the number of trips produced by the site during the PM peak of the adjacent street and the gross floor area of the convenience store. This equation is presented below.

$$
\text { PMTRIPS }=92.8+0.0571 G F A \quad ;
$$

The coefficient of determination for this model is 0.82 and the adjusted $\mathrm{R}^{2}$ is 0.76 . This model is significant at the 0.034 level. Figure 5.1 depicts the "best fit" curve of PMTRIPS vs GFA. 
Figure 5.6 Best Fit Curve PMTRIPS vs GFA

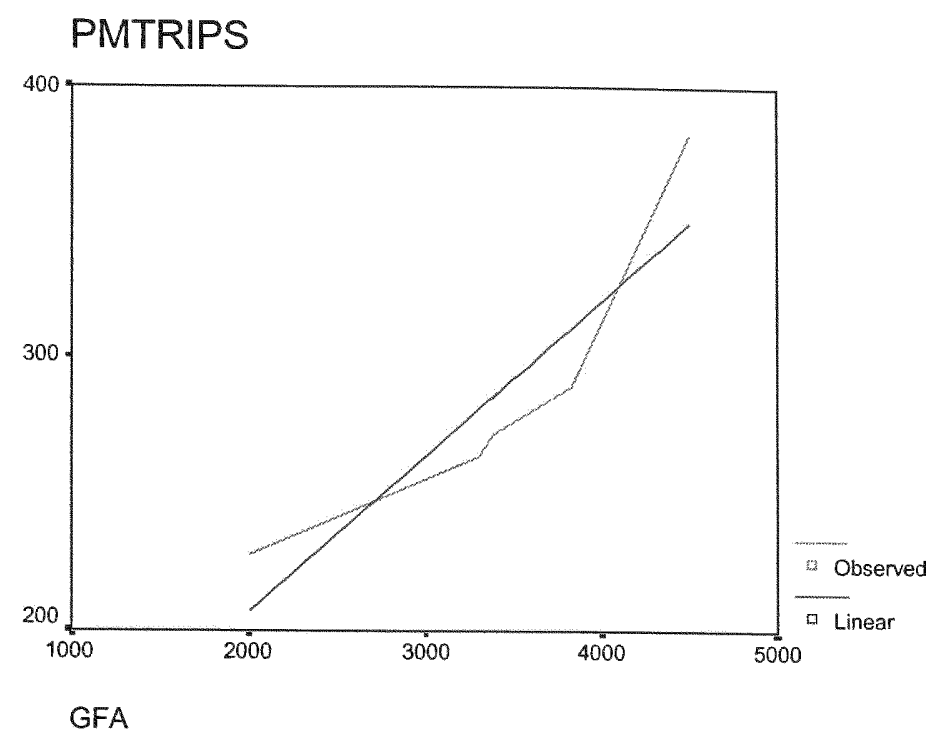

\subsection{DTRIPS Model Using Regression Through the Origin}

Sometimes the regression line is known to go through the origin. This occurs when to a value of $X$ the expected value of $Y$ is zero. The literature review yielded one case in which regression through the origin (no intercept model) was used to estimate PM peak hour of the adjacent street trips. Therefore in this section this method is explored and used to estimate the average weekday trips (DTRIPS) with gross floor area (GFA) as the predictor variable. The regression equation generated using regression through the origin is presented below.

DTRIPS $=1.161 G F A \quad ;$

The coefficient of determination $\left(\mathrm{R}^{2}\right)$ calculated for this regression equation is 0.962 , and the adjusted $R^{2}$ 0.952. This linear regression relationship was found to be significant at the 0.001 level, hence there is only a one percent chance that this relationship is in error. 
As mentioned in chapter two the coefficient of determination found using regression through the origin is not comparable to the intercept model. In regression through the origin the coefficient of determination measures the proportion of the variability in the dependent variable about the origin explained by regression.

\subsubsection{Comment on Regression Through the Origin}

Neter, Wasserman and Kutner, (1983), refer to regression through the origin in the following manner: "like any other model, regression through the origin should be evaluated for aptness. Even when it is known that the regression function must go through the origin, the function might not be linear or the variance of the error terms might not be constant. Often one can not be sure in advance that the regression function goes through the origin, and it is then safe practice to use the general model. If the regression does go through the origin, $b_{\circ}$ will differ from zero only by a small sampling error, and unless the sample size is very small, use of the general, i.e. intercept model, has no disadvantages of any consequence. If the regression does not go through the origin, use of the general model will avoid potentially serious difficulties resulting from forcing the regression through the origin when this is not appropriate." Therefore the use of this model only has a marginal benefit when the sample size is large. On the other hand if the model is applied to a small sample size one must be certain of the validity of the assumption, $\left(b_{0}=0\right)$, otherwise the analysis is not valid. 


\section{CHAPTER 6}

\section{SUMMARY AND CONCLUSION}

\subsection{Introduction}

This study was undertaken to test the validity of the use of ITE's trip generation rates for LU-846 to estimate trips produced by a recently introduced land use in MiamiDade County, the gasoline station with convenience market, quick service restaurant and car wash. But more importantly this research intended to build trip generation models to explain the trip generation characteristics of this new land use rising in popularity in Miami-Dade county and other areas of the United States. In order to build the models, data were collected at five sites representative of the new land use. Multiple regression analysis was performed to generate models for the average daily trips and trips produced during the PM peak hour of the adjacent street. Number of driveways (DRW) and gross floor area of the convenience store (GFA) were used as independent variables. The motivation to build local trip generation models lies in the fact that local trip generation models are more representative of local trip generation characteristics than models built with a wide range of data sources. This study is the first attempt to generate local trip generation models for this type of development in Miami-Dade County.

\subsection{Discussions}

The literature research showed the inherent difficulty in finding a single independent variable that can explain trip generation of gasoline stations of varying design. Furthermore, the literature reviewed also yielded the widespread reliance on only 
two independent variables, vehicle fueling positions and gross floor area of the convenience store to explain the trip generation characteristics of gasoline stations.

In this study as a result of the data analysis a negative linear relationship was found between the average daily traffic and vehicle fueling positions, this is a very significant result. It implies that trip generation decreases with increases in vehicle fueling positions. The implications of this negative linear relationship should be viewed in the context of the new land use. This new type of land use follows a current trend in gasoline station development which increasingly relies on the incorporation of ancillary services such as the convenience market, and the quick service restaurant for its success. In other words, the sale of gasoline may not be the primary business for this type of development. In this context the number vehicle fueling positions may not be as determinant in trip generation as previously thought. Using the same argument gross floor area of the convenience store should be a better trip generation predictor for this type of development. This is consistent with the results of the analysis performed, which shows a high degree of correlation between PM peak hour trips and gross floor area. Moreover, other factors such as the total area of the site is a determinant factor in the number of vehicle fueling positions. For instance if enough land is available for the development, there is a tendency by developers to increase the number of fueling positions in order to provide additional fueling positions to allow for servicing of the pumps as indicated by the owner of one of the sites surveyed. Hence other factors that affect the number of fueling positions are not directly related to attracting vehicles to the site. 
Furthermore, there may be other factors that may have a significant impact on the number of trips produced by gasoline stations and in particular gasoline stations that incorporate a quick service restaurant to the convenience store. For instance, access was not used in the literature reviewed as an independent variable. Access, i.e. number of driveways, was found, in this study, to be correlated with the average daily trips and trips produced during the PM peak of the adjacent street. This is significant since this new variable may be used to build a multiple regression model and thus obtain a model that estimates trips with a greater degree of confidence. Moreover, using number of driveways as an independent variable should not present any difficulties since this variable can easily obtained from the proposed plans for a new site.

Additionally, the market presence of a particular gasoline station brand may influence its trip generation characteristics. For instance one brand of gasoline station may have a greater presence in a particular market and therefore that particular brand is more recognized by consumers. Furthermore, socioeconomic factors may also play a significant role in the trip producing characteristics of this type of development. If one considers, for instance income levels one may argue that a gasoline station with convenience market, quick service restaurant and car wash located within a lower income area may produce more trips than one located within a higher income area. The argument is that a person with a lower income would make many small purchases rather than one single purchase. Incorporating socioeconomic variables in this type of analysis may considerably improve the models. Further research is needed in order to assess the contributions of socioeconomic factors to the trip generation of this type of development. 


\subsection{Conclusion}

The objectives of the research were twofold: (a) validate the use of ITE's LU-846 rates to estimate trip generation of the new land use, and (b) build local trip generation models for gasoline stations with convenience market, quick service restaurant, and car wash. From the analysis performed the following conclusions were reached:

- Trip generation rates for ITE's LU-846 do not accurately estimate trip generation of the newly introduced land use, gasoline station with convenience market, quick service restaurant and car wash.

- Multiple regression models for estimating average daily trips and trips during the PM peak hour of the adjacent street were developed using gross floor are (GFA) and number of driveways (DRW) as the predictor variables. The coefficients of determination for these models were 0.84 and 0.70 respectively. Multiple regression performed on a large sample size provides more reliable results than simple regression analysis.

- Socioeconomic variables such as income levels may have an impact on trip generation of this type of development.

- Gross floor area of the convenience store (GFA) was found to be a better predictor of the number trips produced by the sites than vehicle fueling positions. 


\subsection{Summary of Literature Review}

As stated in the introduction to this chapter, the focus of the literature review for this research centered on two topics: the factors that have been previously identified as significant determinants of injury severity and the statistical modeling approach to estimate the contribution of these factors to injury severity level. The literature yielded three main categories of factors that have been used in previous research to investigate injury severity risk, namely, driver, and road characteristics and environmental conditions. In addition, socioeconomic indicators such as ethnic background and school attainment have also been used as covariates in injury severity research.

The literature also yielded several methodological approaches to investigate the association between these factors and injury severity level. Previous authors have used logistic regression, log-linear models, and econometric models, namely the nested logit model and ordered discrete data models. However, the most recent literature shows a preference towards the used of ordered discrete data models. One significant advantage of using these models is that they account for the ordinal nature of the injury severity levels; this is not the case of the nested logit model. 
Kawamura, J. H. (1993) "Service Station Trip Generation", ITE Journal, March 1993, pp. 23-28.

Keller, R. C., Sherin, J. E. and Connor, M. C. (1992),Trends in Published ITE Trip Generation Rates, Proceedings of the Conference: Site Impact Traffic Assessment, American Society of Civil Engineers, New York, 1992.

Kline, Rex B. (1998), "Principles and Practice of Structural Equation Modeling", The Guilford Press, A Division of Guilford Publications, New York, 1998

Koby, A. L. and McKinstry D. L. (1992),Trip Generation Rates, A Historical Look, Proceedings of the Conference: Site Impact Traffic Assessment, American Society of Civil Engineers, New York, 1992.

Kramer, W. and Sonnberger, H. (1986), "The Linear Regression Model Under Test", Physica-Verlag Heidelberg, Germany, 1986.

Long, G. and Morrison B. (1992), "Trip Generation of Convenience Stores with Gas Pumps", Transportation Research Center, University of Florida, February 1992.

Neter, J., Wasserman, W. and Kutner, M. (1983). Applied Linear Regression Models, Richard D. Irwin, Inc. Homewood, Illinois, (1983)

Rawlings, J. O. (1988). Applied Regression Analysis: A Research Tool, Wadsworth \& Brooks/Cole Advanced Books \& Software, Pacific Grove, California.

Scheaffer, R. L. and McClave, J. T. (1995), "Probability and Statistics for Engineers", Duxbury Press, Wadsworth, Inc., 1995.

SPSS (1997), SPSS Base 7.5 for Windows User's Guide, SPSS Inc. Chicago, Illinois, 1997. 
Tabachnick, B.G. and Fidell, L.S. (1983). Using Multivariate Statistics. Harper \& Row, Publishers, N.Y.

Younger, Mary Sue (1979), A Handbook for Linear Regression, Duxbury Press, North Scituate, Massachusetts, 1979. 